\title{
Los Estados De Afecto Positivo Y Afecto Negativo Como Variables Explicativas De La Ansiedad Escénica En Estudiantes De Magisterio
}

\author{
Francisco Javier Zarza-Alzugaray, Dr. \\ Juan Carlos Bustamante, Dr. \\ Oscar Casanova, Dr. \\ Santos Orejudo, Dr. \\ University of Zaragoza, Spain
}

doi: 10.19044/esj.2016.v12n31p448 URL:http://dx.doi.org/10.19044/esj.2016.v12n31p448

\begin{abstract}
In the students of Primary Education Teaching we see how music performance anxiety proves to be one of the key problems for the overcoming of subjects related to musical study and practice. Thus we see how this problem is related to personal characteristics such as general anxiety, either trait or state, self-perceived musical competence or affects, either positive or negative, both trait and state. In this study we sought to analyse the predictive value that these variables have on the levels of the performance anxiety experienced. The results show how half of the students participating in the study show higher levels of performance anxiety than the theoretical mean of the measuring instrument used and how this construct is significantly related to general anxiety, self-perceived musical ability and affect. It has also been shown how, in order to explain the greater or lesser presence of music performance anxiety, both positive and negative affect in their state measure are those with greater explanatory capacity. More research is needed in order to create specific training programs that help students to generate regulatory adaptive mechanisms that help to better focus the music performance during the evaluative processes of this type of subjects.
\end{abstract}

Keywords: Music education, music performance anxiety, positive affect, negative affect, primary education teachers. 


\section{Resumen}

En estudiantes de magisterio vemos cómo la ansiedad escénica musical resulta ser uno de los problemas clave para la superación de asignaturas relacionadas con el estudio y práctica musical. Así vemos cómo este problema está relacionado con características personales como la ansiedad general, bien rasgo bien estado, la competencia musical autopercibida o los afectos, bien positivos bien negativos, tanto rasgo como estado. En este estudio buscamos analizar el valor predictivo que estas variables tienen sobre los niveles de ansiedad escénica experimentados. Los resultados muestran cómo la mitad de los estudiantes participantes en el estudio presentan valores de ansiedad escénica superiores a la media teórica del instrumento de medida empleado y cómo este constructo está significativamente relacionado con la ansiedad general, la habilidad musical autopercibida y los afectos. También se ha comprobado cómo, de cara a explicar la mayor o menor presencia de ansiedad escénica, tanto el afecto positivo como el negativo en su medida estado son los que mayor capacidad explicativa poseen. Se hace necesaria más investigación de cara a poder crear programas de formación específicos que ayuden a los estudiantes a generar mecanismos adaptativos regulatorios que ayuden a enfocar mejor la actuación en los procesos evaluativos de este tipo de asignaturas.

Palabras clave: educación musical, ansiedad escénica, afecto positivo, afecto negativo, magisterio.

\section{Introducción}

Investigaciones como la de Orejudo et al. (2006) señalan que los estudiantes universitarios se ven expuestos, muy a menudo, a diferentes tipos de estresores y en las etapas de educación superior encontramos que se pueden generar problemas de ansiedad concretos. Así, la ansiedad escénica "es la experiencia de una aprensión ansiosa marcada y persistente relacionada con una actuación musical que se ha presentado a través de vulnerabilidades biológicas y/o psicológicas subyacentes y/o experiencias específicas de ansiedad condicionada...“ (Kenny, 2011: p.61). Tal y como apunta Demirbatir (2012), el conocimiento de la ansiedad y los niveles de estrés en los estudiantes universitarios de música es algo importante ya que son alguno de los problemas psicológicos con más incidencia en esta población. De hecho, en las enseñanzas que reciben los estudiantes de magisterio en las asignaturas relacionadas con la música en la universidad, la ansiedad escénica en los momentos de ejecución pública o evaluación es uno de los aspectos más relevantes de su práctica, pudiendo incluso llegar a convertirse en un problema real de cara a la superación de estas asignaturas. Debemos recordar que la enseñanza-aprendizaje de la música en cualquier 
nivel educativo, también el universitario, conlleva trabajar obligatoriamente una serie de contenidos eminentemente prácticos y de exposición pública.

No es extraño ver en estas etapas, como se apunta en McPherson y McCormick (2006), estudiantes en los que bajos niveles de autoeficacia y bajos niveles de autoestima puedan provocar problemas emocionales de tipo ansioso que generan estrés y ansiedad anticipatoria ante los exámenes; más aun los que implican una actuación pública como pueda ser una interpretación musical en la que el proceso de ejecución no admita posibilidad de enmendar errores. En ocasiones, estos problemas pueden estar detrás no solo del detrimento de la habilidad interpretativa sino incluso en el abandono de las asignaturas durante años o, en casos extremos, el abandono de los estudios universitarios.

Contemplando las posibles respuestas o consecuencias que la ansiedad escénica puede tener en las personas que la sufren, investigaciones como las de Herrera y Jorge (2013) o Papageorgi, Hallam y Welch (2007), determinan tres dimensiones diferenciadas. Una primera dimensión de corte fisiológico, en la que es habitual sentir un incremento en las pulsaciones del corazón, necesidad de orinar, sudoración excesiva, temblores de manos, piernas y de la voz, náuseas o incluso vómitos, entre los más frecuentes. La segunda referida a problemas cognitivos; encontramos como tales fallos de memoria o incapacidad para razonar de manera adaptativa para una resolución de la tarea satisfactoria, entre otros. En la tercera, correspondiente al plano conductual, las reacciones más frecuentes suelen ser la presencia de conductas de evitación o incluso de escape de la situación ansiógena.

Para Wilson (2002), existen distintos factores que en su interacción pueden ser capaces de explicar la mayor o menor presencia de ansiedad específica, en este caso ansiedad escénica. Así, explica que la ansiedad general, tanto rasgo como estado y el nivel de dominio conseguido sobre la música a interpretar, están detrás de mayores o menores niveles de ansiedad escénica.

Sin embargo, vemos en investigaciones como la de Zarza, Casanova y Robles (2016) cómo los problemas de ansiedad escénica no han recibido especial atención entre la comunidad de estudiantes universitarios españoles; si bien hay investigaciones que consideran a la ansiedad como uno de los principales problemas psicológicos de la actualidad (Guillén y Buela, 2011). Además, los pocos estudios que encontramos se centran en estudiantes con un marcado carácter profesionalizante en la ejecución musical como puedan ser los estudiantes de conservatorios, mientras que no existe un corpus de referencia en lo que al estudio de las habilidades interpretativas se refiere de los estudiantes de los Grados en Magisterio, tanto en Educación Infantil como en Educación Primaria. 
A su vez y estrechamente relacionado con la ansiedad encontramos el aspecto afectivo de la persona. Así, para Páez y Carbonero (1993) la afectividad engloba tanto las emociones como los estados de ánimo y las evaluaciones afectivas. Además, y según Stincer y Monroy (2012) a lo anterior se le pueden sumar las respuestas de carácter fisiológico o somático y las reacciones cognitivas que estos estados desencadenan. Igualmente, y tal y como sugieren Watson y Clark (1994), son dos las grandes estructuras afectivas, el afecto positivo y el negativo. De esta forma, las personas con un afecto positivo alto presentan una alta energía en la realización de tareas, altas tasas de concentración y agrado por la dedicación en la resolución de tareas; mientras que las personas con un afecto positivo bajo son descritas por estados de tristeza y letargo. En el caso del afecto negativo, las personas con niveles elevados del mismo vemos que presentan distintos estados de ánimo caracterizados por la culpa, nerviosismo, ira o temor. En el caso de niveles bajos de afecto negativo encontramos que son característicos los estados emocionales calmados y serenos (Cazalla-Luna y Molero, 2014).

Por tanto, con esta investigación buscamos realizar las primeras aproximaciones al estudio del valor predictivo que variables como la ansiedad rasgo y la ansiedad estado, el afecto positivo y negativo, así como el nivel de dominio percibido, pueden tener sobre la ansiedad escénica percibida por estudiantes universitarios españoles. Para ello, se plantea realizar análisis estadísticos descriptivos y multivariados que nos permitan determinar, de forma exploratoria, cómo se comportan los datos en este tipo de muestra respecto a las variables estudiadas y la relación entre las mismas.

\section{Método}

\section{Participantes}

La muestra de este estudio estaba compuesta por 33 estudiantes de tercer curso del Grado en Magisterio en Educación Primaria de la Universidad de Zaragoza (22 chicas, 8 chicos y 3 casos considerados perdidos) de entre 20 y 26 años de edad (\rceil$=21.26$; D.T. $=1.77)$. Todos ellos cursaban la asignatura obligatoria Fundamentos de Educación Musical en la Facultad de Ciencias Humanas y de la Educación.

\section{Procedimiento}

Dados los objetivos planteados en el estudio, se decidió realizar la recogida de los datos, en un único punto temporal, de forma grupal. En este sentido, se recogen medidas de habilidad musical percibida, niveles de ansiedad estado y rasgo, niveles de ansiedad escénica, así como niveles de afecto positivo y negativo, tanto estado como rasgo, inmediatamente antes de que cada uno de los estudiantes se enfrentara a una prueba en la que se les exigía la realización de distintos ejercicios musicales (rítmicos, melódicos y 
rítmico-melódicos) en audiencia pública. La realización de estos ejercicios son necesarios en esta asigntatura para su superación.

\section{Instrumentos y análisis de datos}

El método de evaluación consistió en la compilación en un instrumento ad hoc en el que se recogían las distintas variables objeto de estudio mediante técnicas de auto-informe. Así se empleó un instrumento adaptado por Zarza y Zarza (2016) que permitía evaluar tanto la habilidad musical autopercibida (12 ítems; $\left.\alpha_{\text {Cronbach }}=.826\right)$ como la ansiedad escénica en la misma por parte de los estudiantes ( 3 ítems; $\alpha_{\text {Cronbach }}=.765$ ) medidos con una escala Likert de 5 puntos en las que puntuaciones altas implican características personales elevadas en los constructos evaluados. Además se emplearon diferentes cuestionarios asentados en la comunidad científica encargados de evaluar la ansiedad estado y rasgo (STAI-R y STAI-T) de Spielberger et al. (1983), el afecto positivo y negativo (PANAS-ESTADO y PANAS-RASGO) de Watson, Clark y Tellegen (1988) y una pregunta de carácter sociodemográfico para conocer el sexo de los estudiantes.

En lo concerniente al tipo de análisis de los datos obtenidos a partir de los cuestionarios anteriormente indicados, decir que se realizó empleando el programa estadístico SPSS 19.0 y que se llevaron a cabo ANOVA, análisis correlacionales y regresiones.

\section{Resultados}

Una primera aproximación a las distribuciones de las escalas muestra cómo todas ellas responden de acuerdo a una distribución normal de los datos aportados por los estudiantes, ya que las pruebas Kolmogorov-Smirnov para contrastar la hipótesis nula de normalidad obtuvieron todas ellas $p$ values mayores de 05 .

En lo que concierne a la distribución muestral de la variable criterio estudiada (ansiedad escénica musical), se ha obtenido una media igual a 9.60 $(D . T .=2.60)$ sin que exista una diferencia de medias significativas $(t=1.031$; $\mathrm{p}=.316)$ con respecto a la media teórica del instrumento (9 puntos). Además, vemos cómo un 35\% de los estudiantes se sitúan en puntuaciones menores que la media teórica del instrumento (9 puntos), y un 15\% se sitúa justo en la media teórica dejando un 50\% de estudiantes que puntúan por encima de la media teórica del instrumento de medida.

Por su parte, respecto de las comparaciones de medias en las puntuaciones en función del sexo de los estudiantes, vemos cómo en el caso de la escala de ansiedad escénica específica, se dan diferencias de medias significativas $\left(F_{1,16}=4.653 ; p=.047 ; \eta^{2}=.225\right)$, siendo mayor la puntuación en las chicas (10.38) que en los chicos (7.60), con un tamaño del efecto considerable del sexo del $22.5 \%$. 
Tabla 1. ANOVA. Variable Criterio: Ansiedad Escénica.

\begin{tabular}{ccccccc}
\hline Sexo & Media & Desv. típ. & N & F & Sig. & $\eta^{2}$ \\
\hline Chica & 10.38 & 2.46 & 13 & \multirow{2}{*}{4.653} & .047 & .225 \\
Chico & 7.60 & 2.40 & 5 & & & \\
\hline
\end{tabular}

Sin embargo, en el resto de variables contempladas en este estudio no se han obtenido diferencias significativas ( $p>.05)$ de puntuaciones medias en función del sexo de los estudiantes.

En el caso de las relaciones entre las distintas variables vemos cómo un análisis correlacional indica que la ansiedad específica está correlacionada inversamente con la habilidad musical autopercibida ( $\mathrm{r}=-.458 ; \mathrm{p}=.042)$ así como de manera directamente proporcional con la ansiedad estado $(\mathrm{r}=.656$; $\mathrm{p}=.015)$. A su vez también con el afecto negativo tanto estado ( $\mathrm{r}=.685$; $\mathrm{p}=.001)$ como rasgo $(\mathrm{r}=.500 ; \mathrm{p}=.025)$. Vemos igualmente cómo existen correlaciones significativas $(\mathrm{r}=.863 ; \mathrm{p}=.000)$ entre la ansiedad rasgo y la ansiedad estado, entre la ansiedad rasgo y el afecto negativo estado $(r=.529$; $\mathrm{p}=.020)$, y entre la ansiedad rasgo y el afecto negativo rasgo $(\mathrm{r}=.659$; $\mathrm{p}=.001$ ). Además, comprobamos cómo también existen correlaciones significativas entre la ansiedad estado y el afecto negativo $(r=.885 ; \mathrm{p}=.000)$ y la ansiedad estado y el afecto negativo rasgo $(\mathrm{r}=.642$; $\mathrm{p}=.018)$. Se comprueba asimismo cómo el afecto positivo estado correlaciona con el afecto positivo rasgo ( $\mathrm{r}=.586 ; \mathrm{p}=.007)$, así como el afecto negativo correlaciona con el afecto negativo rasgo $(\mathrm{r}=.724 ; \mathrm{p}=.000)$.

Tabla 2. Correlaciones.

\begin{tabular}{cccccccc}
\hline & A.E. & Habilidad_musical & Ansiedad_E & AP_E & AN_E & AP_R & AN_R \\
\hline A.E. & 1 & -.458 & .656 & & .685 & & .500 \\
\hline Ansiedad_R & & & .863 & & .529 & & .659 \\
\hline Ansiedad_E & & 1 & & .885 & & .642 \\
\hline AP_E & & & 1 & & .586 & \\
\hline AN_E & & & & 1 & & .724 \\
\hline AP_R & & & & & & 1 \\
\hline AN_R & & & & & & & \\
\hline
\end{tabular}

Llegados a este punto consideramos útil, a tenor de las correlaciones anteriormente comentadas, plantear un modelo de regresión que sea capaz de explicar parte de la varianza del factor específico de la ansiedad escénica de los estudiantes.

Así, empleando la técnica de regresión de pasos sucesivos (step wise) vemos cómo obtenemos un modelo final significativo $(\mathrm{F}=7.657$; $\mathrm{p}=.013$; $\mathrm{D}$ $\left.\mathrm{W}=2.672<\mathrm{D}-\mathrm{W}_{\mathrm{S}-\mathrm{W} ; \mathrm{n}=11 ; \mathrm{K}=6 ; \mathrm{p}<.05}=3.004\right)$ que es capaz de explicar el $66.6 \%$ de la varianza del factor específico de ansiedad $\left(\mathrm{R}^{2}=.666\right)$ a partir de la acción 
significativa y directa del afecto negativo estado $(\beta=.602 ; \mathrm{t}=3.068 ; \mathrm{p}=.018) \mathrm{e}$ inversa del afecto positivo estado $(\beta=-.455 ; \mathrm{t}=-2.429 ; \mathrm{p}=.045)$.

Tabla 3. Modelo de regresión. Variable criterio: Ansiedad Escénica.

\begin{tabular}{ccccccc}
\hline Regresores & $\beta$ & $\mathrm{t}$ & $\mathrm{Sig}$. & $\mathrm{R}^{2}$ & $\mathrm{~F}_{3,7}$ & Sig. \\
\hline AN_E & .602 & 3.068 & .018 & \multirow{2}{*}{666} & \multirow{2}{*}{7.657} & \multirow{2}{*}{.013} \\
AP_E & -.455 & -2.429 & .045 & & & \\
\hline
\end{tabular}

Vemos, además, cómo el resto de variables incluidas en el modelo de regresión no tienen capacidad explicativa significativa en el modelo. Así, el sexo ( $\mathrm{t}=-1.449 ; \mathrm{p}=.190)$, la habilidad musical autopercibida ( $\mathrm{t}=-1.034$; $\mathrm{p}=.341)$, la ansiedad-rasgo ( $\mathrm{t}=-2.367 ; \mathrm{p}=.056)$, el afecto-positivo-rasgo ( $\mathrm{t}=-$ .293; $\mathrm{p}=.779)$, el afecto-negativo-rasgo $(\mathrm{t}=-.282 ; \mathrm{p}=.787)$ y la ansiedad estado ( $\mathrm{t}=-1.356 ; \mathrm{p}=.224)$.

\section{Conclusión}

A tenor de los resultados anteriormente expuestos podemos decir que la ansiedad escénica es un problema presente en los estudiantes participantes en el estudio. Así, hemos comprobado cómo la mitad de los estudiantes presentan valores por encima de la media y un $10 \%$ de ellos presentan puntuaciones de más de una desviación típica por encima de la media. Estas cifras concuerdan con otras investigaciones similares como la de Zarza, Casanova y Orejudo (2016), o Steptoe (2001).

Asimismo, el hecho de que las chicas reporten mayores niveles de ansiedad escénica que los chicos también ha sido descrito con anterioridad por Kenny, Davies y Oates (2004) o Kaspersen y Götestam (2002).

Sin embargo, y parcialmente distinto a lo que Wilson (2002) aduce, la ansiedad rasgo de los estudiantes no ha presentado capacidad correlacional ni tampoco explicativa de las variaciones en la ansiedad escénica. Si bien, las características personales relacionadas con los niveles de ansiedad más inmediatos y anteriores a la actuación (ansiedad estado) han presentado capacidad correlacional con la ansiedad escénica.

Debemos apuntar como significativo el hecho de que los predictores más potentes que se han encontrado en este estudio hayan sido las características personales de afecto, tanto negativo como positivo, referidas en su versión estado. Hay que tener en cuenta que se ha encontrado relación positiva y negativa entre el afecto negativo y positivo (en su versión estado) y niveles de ansiedad, respectivamente (Merz y Roesch, 2011). Así, la ansiedad escénica es una reacción psicofisiológica que puede acompañar a la experiencia afectiva negativa que se produce ante la situación estresante de la actuación (Kenny, 2011). De hecho, estados de afectividad negativa se relacionan con el estrés más que estados de afectividad positiva (Polk et al., 2005; Merz y Roesch, 2011). En este sentido, la afectividad positiva se ha 
relacionado con mayores niveles del conocido estado "flow" (Heller, Bullerjahn y von Georgi, 2015). Este estado implica una absoluta absorción en la tarea realizada sin necesidad de realizar un gran esfuerzo para concentrarse en ella, y representa un estado óptimo donde recursos y demandas se encuentran compensadas alejándose de la experiencia de estrés (Csikszentmihalyi, 2010; Heller, Bullerjahn y von Georgi, 2015). Por tanto, puede que aquellos estudiantes que refieran mayores niveles de afectividad positiva perciban la situación como menos estresantes, y más controlada, y eso les permita experimentar una menor ansiedad escénica.

A su vez, debemos tener en cuenta que una limitación importante para este estudio ha sido fundamentalmente el tamaño muestral y la pérdida de información a la hora de completar los cuestionarios de auto-informe. Este hecho hace necesario, si cabe, seguir profundizando en las relaciones entre estos constructos de cara a explicar la ansiedad escénica y cómo pueden mejorarse las habilidades musicales de cara a una mejor preparación de las asignaturas. La necesidad de plantear iniciativas que promuevan los estados de "flow" en los propios alumnos, y otros estados positivos placenteros (optimismo...), se hace más que fehaciente para hacer frente a las demandas exigidas por las situaciones de ejecución/interpretación musical. De esta forma, se buscaría promover condiciones idóneas que redunden en un mayor rendimiento académico.

\section{References:}

1. Cazalla-Luna, N. \& Molero, D. (2014). Inteligencia emocional percibida, ansiedad y afectos en estudiantes universitarios. Revista Española de Orientación y Psicopedagogía, 25(3), 56-73.

2. Demirbatir, R. E. (2012). Undergraduate music students' depression, anxiety and stress levels: a study from Turkey. Procedia - Social and Behavioral Sciences, 46, 2995-2999.

3. Csikszentmihalyi, M. (2010). Flow. Das Geheimnis des Glücks (15th Ed.). Stuttgart: Klett-Cotta.

4. Guillén, A. \& Buela, G. (2011). Actualización psicométrica y funcionamiento diferencias de los ítem en el State Trait Anxiety Inventory (STAI). Psicothema, 23(3), 510-515.

5. Heller, K., Bullerjahn, C. \& von Georgi, R. (2015). The relationship between personality traits, flow-experience, and different aspects of practice behavior of amateur vocal students. Frontiers in Psychology, 6, 1901. doi:10.3389/fpsyg.2015.01901

6. Herrera, L. \& Jorge, G. (2013). Ansiedad escénica musical en estudiantes de flauta travesera. Eufonía. Didáctica de la música, 57, 43-55. 
7. Kaspersen, M. \& Götestam, K. G. (2002). A survey of Music performance anxiety among Norwegian music students. European Journal of Psychiatry, 16(2), 69-80.

8. Kenny, D. T. (2011). The psychology of music performance anxiety. Oxford, UK: Oxford University Press.

9. Kenny, D. T., Davis, P. \& Oates, J. (2004). Music performance anxiety and occupational stress amongst opera chorus artists and their relationship with state and trait anxiety and perfectionism. Journal of Anxiety Disorders, 18(6), 757-777. doi:10.1016/j.janxdis.2003.09.004

10. McPherson, G. E. \& McCormick, J. (2006). Self- Efficacy and music performance. Psychology of Music, 34(3), 322-336. doi:10.1177/0305735606064841

11. Merz, E. L. \& Roesch S. C. (2011). Modeling trait and state variation using multilevel factor analysis with PANAS daily diary data. Journal of Research in Personality, 45(1), 2-9. doi:10.1016/j.jrp.2010.11.003

12. Orejudo, S., Nuño, J., Fernández, T., Ramos, M. T. \& Herrero, M. L. (2006). Participación del alumnado universitario en el aula. Una investigación sobre el temor a hablar en público en grandes grupos. Revista de Psicología General y Aplicada, 59, 257-276.

13. Páez, D. \& Carbonero, A. (1993). Afectividad, Cognición y Conducta social. Psicothema, 5, 133-150.

14. Papageorgi, I., Hallam, S. \& Welch, G. (2007). A conceptual framework for understanding musical performance anxiety. Research Studies in Music Education, 28, 83-107. doi:10.1177/1321103X070280010207

15. Polk, D. E., Cohen, S., Doyle, W. J., Skoner, D. P. \& Kirschbaum, C. (2005). State and trait affect as predictors of salivary control in healthy adults. Psychoneuroendocrinology, 30, 261-272.

16. Spielberger, C. D., Gorsuch, R. C., Lushene, R. E., Vagg, P. R. \& Jacobs, G. A. (1983). Manual for the State-Trait Anxiety Inventory. Palo Alto, California (USA): Consulting Psychologists Press.

17. Steptoe, A. (2001). Negative emotions in music making: The problem of performance anxiety. In P. N. Juslin \& J. A. Sloboda (Eds.), Music and emotion: Theory and research (pp. 291-307). Oxford, UK: Oxford University Press.

18. Stincer, D. \& Monroy, Z. (2012). Los afectos en la argumentación científica: una útil perspectiva para la formación de la habilidad de argumentar. Revista Electrónica Nova Scientia, 4(2), 110-128.

19. Watson, D. \& Clark, L. A. (1994). The PANAS-X. Manual for the positive and negative affect schedule. Iowa City, Iowa (USA): University of Iowa. 
20. Watson, D., Clark, L. A. \& Tellegen, A. (1988). Development and validation of brief measures of positive and negative affect: The PANAS Scales. Journal of Personality and Social Psychology, 47, 1063-1070.

21. Wilson, G. D. (2002). Psychology for performing artists (2nd Ed.). London: Whurr.

22. Zarza, F. J., Casanova, O. \& Orejudo, S. (2016). Ansiedad escénica en estudiantes de piano. Ulu. Revista científica sobre la imaginación, 1, 28-35.

23. Zarza, F. J., Casanova, O. \& Robles, J. (2016). Relación entre ansiedad escénica, perfeccionismo y calificaciones en estudiantes del Título Superior de Música. ReiDoCrea. Revista electrónica de investigación y Docencia Creativa, 5, 16-21.

24. Zarza, F. J. \& Zarza, M. B. (2016). Una explicación desde la creatividad de la competencia musical en Educación Primaria. Ulu. Revista científica sobre la imaginación, 1, 36-41. 